\title{
INVOLUTIONS OF GRAPH LINK EXTERIORS WHOSE FIXED POINT SETS ARE CLOSED SURFACES
}

\author{
TORU IKEDA
}

\author{
(Received 24 June 2009)
}

\section{Abstract}

A link $L$ in $S^{3}$ possibly admits an involution of the exterior $E(L)$ with fixed point set a closed surface, which is not extendable to an involution of $S^{3}$. In this paper, we focus on the case of graph links and show that the genus of the surface provides a lower estimate of the number of link components.

2000 Mathematics subject classification: primary 57M25; secondary 55M35.

Keywords and phrases: graph link, involution, fixed point set, closed surface.

\section{Introduction}

Symmetries of links $L$ in $S^{3}$, such as periodicity, strong invertibility and strong amphicheirality, are defined by using periodic maps $f$ (orientation-preserving or not) of $S^{3}$ (see [2]). The fixed point set of $f$ is a closed surface if and only if $f$ is a reflection in a sphere. However, the exterior $E(L)$ of $L$ possibly admits an involution with fixed point set a nonspherical closed surface. For example, the twocomponent link $L$ illustrated in Figure 1(1) admits an involution of $E(L)$ with fixed point set a torus, which interchanges the boundary components so that a meridian of a component is carried to a longitude of the other (see [5]). This is verified by the satellite construction illustrated in Figure 1(2), where the involution of $E(L)$ induces involutions of the exteriors of the pattern and the companion. If a link $L$ has more than two components, $E(L)$ possibly admits an involution $f$ with fixed point set a closed surface $F$ of negative Euler characteristic. In this paper, we prove the following theorem.

THEOREM 1.1. If the exterior of a nonsplittable graph link $L$ in $S^{3}$ admits an involution with fixed point set a closed surface $F$ of genus $g(F)$, then $L$ has at least $2 g(F)$ components.

In Section 2 we present a method for constructing a tree with numbered and coloured vertices for a specific submanifold of $E(L)$ which is setwise invariant under the involution. Section 3 is devoted to the proof of Theorem 1.1.

(C) 2010 Australian Mathematical Publishing Association Inc. 0004-9727/2010 \$16.00 


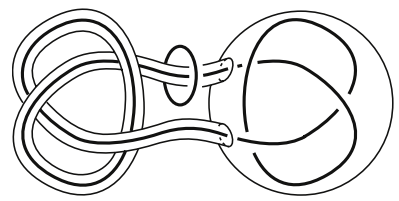

(1)

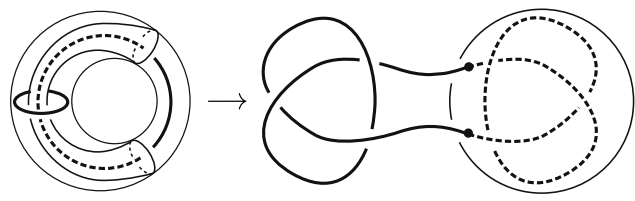

(2)

FIGURE 1. A link and a torus of fixed points.

\section{Tree presentation with numbered and coloured vertices}

Let $L$ be a nonsplittable graph link, and $f$ an involution of the exterior $E(L)$ of $L$ with fixed point set a closed surface $F$ of negative Euler characteristic. We may assume by [9, Theorem 8.6] that the Jaco, Shalen and Johannson (JSJ) system $\mathcal{T}$ of $E(L)$, which is a system of tori given by the JSJ decomposition (see $[6,8]$ ), is (setwise) $f$ invariant. For each $f$-invariant piece $M_{i}$, we may assume by [9, Theorem 2.2] that $f$ preserves the Seifert fibration of $M_{i}$. For each piece $M_{i}$ which is not $f$-invariant, we may assume by [7, Theorem VI.18] that $f$ takes the Seifert fibration of $M_{i}$ onto the Seifert fibration of $f\left(M_{i}\right)$. Then $F$ intersects each piece in a vertical or horizontal surface if $F$ is not disjoint from the piece. Note that a piece is $f$-invariant if and only if it intersects $F$. Denote by $M$ the submanifold of $E(L)$ consisting of the pieces intersecting $F$. Since the orders of the exceptional fibres in each piece in $M$ are pairwise coprime (see [3, Theorem 2]), $f$ cannot interchange them. Moreover, since $f$ is orientation-reversing, $f$ cannot restrict to an involution on a fibred regular neighbourhood of an exceptional fibre. Therefore every piece in $M$ is an $n$-fold composing space, where $n \geq 2$. Note that we can find a composing space not only in the exterior of a composite link but also in the exterior of a link with parallel copies of a component or a link with a cable around a component (see [3, 4]).

Denote by $\Gamma$ the JSJ graph of $M$, where the vertices and edges of $\Gamma$ respectively correspond to the pieces and the tori of the JSJ decomposition of $M$. Colour each vertex in white if $F$ intersects the corresponding piece in a vertical surface, and in black otherwise. For each vertex $v_{i}$ corresponding to a piece $M_{i}$, denote by $\varphi\left(v_{i}\right)$ the number of tori in $M_{i} \cap \partial M$. Then $\varphi\left(v_{i}\right)$ is an even integer such that $d\left(v_{i}\right)+\varphi\left(v_{i}\right) \geq 3$, where $d\left(v_{i}\right)$ is the degree of $v_{i}$, and that $\varphi\left(v_{i}\right)=0$ if $v_{i}$ is black.

If there is an edge joining two white vertices, the fibrations of the corresponding pieces agree (up to isotopy) on the separating torus, which contradicts the property of the JSJ decomposition. If there is an edge joining two black vertices, the restriction of $f$ on the corresponding torus $T$ is impossible since $f$ induces a nontrivial involution on $H_{1}(T)=\mathbb{Z} \oplus \mathbb{Z}$ with three distinct eigenvectors given by $F \cap T$ and the fibres of the pieces on the both sides. Therefore every edge joins a white vertex and a black vertex.

The tree $\Gamma$ obtained as above is call a tree presentation for $M$. For example, Figure 2(1) illustrates a graph link $L$ and a surface $F$ such that $E(L)$ admits an involution with fixed point set $F$. Figure 2(2) illustrates the tree presentation for $E(L)$. 


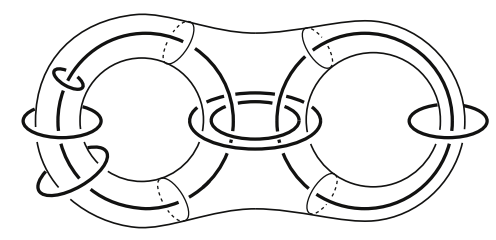

(1)

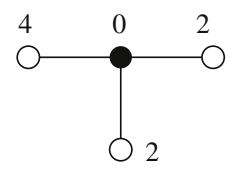

(2)

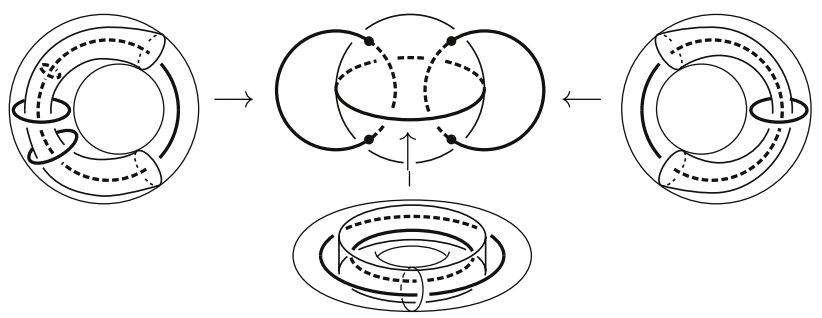

(3)

FIGURE 2. A graph link, a surface of fixed points and the tree presentation.

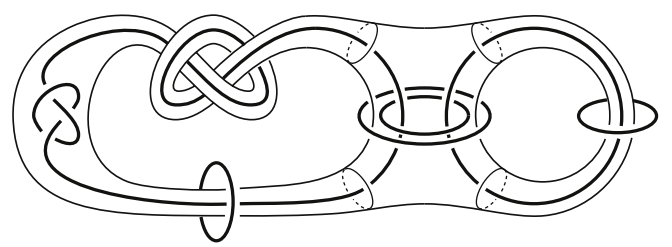

FIGURE 3. A graph link and a surface of fixed points realizing the tree presentation in Figure 2.

We can obtain $L$ and $F$ by the satellite construction illustrated in Figure 2(3) which is the converse of the JSJ decomposition of $E(L)$.

\section{Number of link components}

In the setting of Section 2, the number of components of $L$ is possibly different from the number of the boundary components of $M$. For example, if $L$ is the link illustrated in Figure 3, $M$ is homeomorphic to the exterior of the link illustrated in Figure 2. In this section, we show that the genus of $F$ provides a lower estimate of the number of components of $L$.

Let $T$ be a torus in $S^{3}$ bounding a solid torus $V$. A preferred longitude of $T$ is a longitude which is null-homologous in $S^{3}-$ int $V$.

LEMMA 3.1. Let $M$ be an $n$-fold composing space in $S^{3}$, where $n \geq 2$.

(1) Suppose that a torus $T$ in $\partial M$ bounds a knotted solid torus $V$ which contains $M$. Then if the fibration on $T$ is meridional, at least one of the tori in $\partial M-T$ bounds a knotted solid torus outside $M$, and otherwise every torus in $\partial M-T$ bounds a knotted solid torus outside $M$. 
(2) There is a torus $T$ in $\partial M$ which bounds a solid torus outside $M$ so that the fibration on $T$ is meridional or (possibly not preferred) longitudinal.

Proof. Part (1). Let $D$ be a meridian disk of $V$ which intersects $\partial M-T$ transversally in essential loops.

Assume that the fibration on $T$ is meridional. Since $T$ is incompressible in $M$, $D \cap(\partial M-T)$ is not empty. Suppose that an innermost loop in $D \cap(\partial M-T)$ lies on a torus $T^{\prime}$ in $\partial M-T$. Since $T^{\prime}$ is incompressible in $M, T^{\prime}$ bounds a solid torus $V^{\prime}$ outside $M$. Since the core of $V^{\prime}$ is a satellite of the core of $V, V^{\prime}$ is knotted.

Assume that the fibration on $T$ is not meridional. Since every torus $T^{\prime}$ in $\partial M-T$ contains fibres, $D \cap T^{\prime}$ is not empty. Therefore the conclusion follows by the same argument as above.

Part (2). Suppose that $\partial M$ consists of tori $T_{1}, \ldots, T_{n+1}$. Denote by $V_{i}$ the solid torus in $S^{3}$ bounded by $T_{i}$, which is either a knotted solid torus containing $M$ or a (possibly unknotted) solid torus outside $M$.

Assume that some $V_{i}$ contains $M$. Then if the fibration on $T_{i}$ is meridional, the proof of (1) implies that some another $V_{j}$ is a knotted solid torus outside $M$ where the fibration on $T_{j}$ is meridional. If the fibration on $T_{i}$ is not meridional, the fibration of $M$ extends to a fibration of $V_{i}$, where any other $V_{j}$ but possibly one is endowed with a trivial fibration. Therefore the fibration on $T_{j}$ is (possibly not preferred) longitudinal.

Assume that no $V_{i}$ contains $M$. A result of Burde and Murasugi [1] implies that $M$ is regarded as the exterior of an $(n+1)$-component link $L$ consisting of fibres of some Seifert fibration of $S^{3}$, including the singular fibration corresponding to the circle action on $S^{3}$ with a circle of fixed points. Then $L$ has at least one component $l$ which is a regular fibre. Hence, the conclusion follows from the fact that the fibration around $l$ is (preferred or not preferred) longitudinal.

Proof OF ThEOREM 1.1. It is enough to consider the case $g(F)>1$. Let $f$ be an involution as in the theorem. We may assume that $f$ leaves the JSJ system of the exterior $E(L)$ of $L$ invariant and takes the fibration of each piece onto the fibration of the corresponding piece. Let $M$ be a submanifold of $E(L)$ consisting of the pieces intersecting $F$. For the tree presentation $\Gamma$ of $M$, denote by $\beta(\Gamma)$ the number of black vertices of $\Gamma$. Then $g(F)>1$ implies $\beta(\Gamma)>0$. The proof proceeds by induction on $\beta(\Gamma)$.

Assume that $\beta(\Gamma)=1$. Then $\Gamma$ is a star graph with a black vertex of degree $d \geq 3$ and $d$ white vertices of degree one. Suppose that $M$ consists of pieces $M_{0}, \ldots, M_{d}$ where $M_{0}$ is the $(d-1)$-fold composing space corresponding to the black vertex. Since $F$ intersects $M_{0}$ in two copies of a compact planar surface $F_{0}$ of Euler characteristic $\chi\left(F_{0}\right)=2-d$, we have $\chi(F)=4-2 d$ and therefore $g(F)=d-1$. Each white vertex corresponds to at least 2-fold composing space and therefore $M$ has at least $2 d$ boundary tori. Let $1 \leq i \leq d$. Denote by $T_{i}$ the torus separating $M_{i}$ and $M_{0}$. If a component $S$ of $\partial M \cap M_{i}$ does not separate the components of $L, S$ bounds a nontrivial knot exterior (the exterior of a companion of $L$ ) outside $M$ and so is $f(S)$. Therefore each of $S$ and $f(S)$ bounds a knotted solid torus in $S^{3}$ which 
contains $M$. Lemma 3.1 implies that $M_{i}$ has a meridional fibration on $S \cup f(S)$, and that there is a torus $S^{\prime}$ in $\partial M_{i}-S \cup f(S)$ which bounds a knotted solid torus outside $M_{i}$. Assume that $S^{\prime} \neq T_{i}$. Then $S^{\prime}$ separates at least one component of $L$ from the others and so does $f\left(S^{\prime}\right)$. Then $T_{i}$ separates at least two components of $L$ from the others. Assume that $S^{\prime}=T_{i}$. Then $T_{i}$ possibly does not separate the components of $L$. Since the fibration of $M_{i}$ is meridional on $T_{i}$, the fibration of $M_{0}$ is not. Lemma 3.1 implies that any torus in $\partial M_{0}-T_{i}$ bounds a knotted solid torus outside $M_{0}$. Therefore each $\partial M \cap M_{j}$ with $j \neq i$ contains two tori, which are interchanged by $f$, and each of which bounds a solid torus in $S^{3}$ outside $M$. Hence, each $T_{j}$ separates at least two components of $L$ from the others. Repeating the same argument for all $i, L$ has at least $2 d-2=2 g(F)$ components.

Assume that the theorem is true for $1 \leq \beta(\Gamma)<n$ and consider the case $\beta(\Gamma)=n$. Then $\Gamma$ has a white vertex $w$ of degree $d>1$. Denote by $\mathcal{T}_{w}=T_{1} \cup \cdots \cup T_{d}$ the system of tori corresponding to the edges incident to $w$. Suppose that $\mathcal{T}_{w}$ splits $M$ into $N_{0}, \ldots, N_{d}$, where $N_{0}$ corresponds to $w$, and where $T_{i}$ separates $N_{0}$ and $N_{i}$ for $1 \leq i \leq d$. There are two possibilities: either (1) $T_{i}$ bounds a (possibly unknotted) solid torus $V_{i}$ outside $N_{i}$, or (2) $T_{i}$ bounds a knotted solid torus $V_{i}$ containing $N_{i}$. Moreover, case (2) is divided into three subcases: $F \cap T_{i}$ is either (2a) meridional, (2b) longitudinal, or (2c) nonmeridional and nonlongitudinal.

In case (1), let $L_{i}$ be the link which consists of the components of $L$ outside $V_{i}$, the core of $V_{i}$, and a loop obtained by pushing a component of $F \cap T_{i}$ into int $V_{i}$. Then $E\left(L_{i}\right) \cap V_{i}$ is a 2-fold composing space. Therefore $\left.f\right|_{N_{i}}$ extends to an involution of $E\left(L_{i}\right)$ with fixed point set a closed surface $F_{i}$ which meets $E\left(L_{i}\right) \cap V_{i}$ in an essential annulus.

In case (2a), let $L_{i}=h_{i}\left(L \cap V_{i}\right)$, where $h_{i}$ is a homeomorphism which takes $V_{i}$ onto a regular neighbourhood $W$ of the square knot. Then $\left.h_{i} \circ f\right|_{N_{i}} \circ h_{i}^{-1}$ is an involution on $h_{i}\left(N_{i}\right)$ which extends to an involution of $h_{i}\left(N_{i}\right) \cup\left(S^{3}-\right.$ int $\left.W\right)$ with fixed point set a closed surface $F_{i}$ which meets $S^{3}-$ int $W$ in an essential annulus.

In case (2b), let $L_{i}=h_{i}\left(L \cap V_{i}\right)$, where $h_{i}$ is a homeomorphism which takes $V_{i}$ onto an unknotted solid torus $W$ such that the image of $F \cap T_{i}$ is a parallel copies of a preferred longitude. Then the involution $\left.h_{i} \circ f\right|_{N_{i}} \circ h_{i}^{-1}$ of $h_{i}\left(N_{i}\right)$ extends to an involution of $h_{i}\left(N_{i}\right) \cup\left(S^{3}-\right.$ int $\left.W\right)$ with fixed point set a closed surface $F_{i}$ which meets $S^{3}-$ int $W$ in a parallel copy of a meridian disk.

In case (2c), let $L_{i}$ be the union of $h_{i}\left(L \cap V_{i}\right)$, where $h_{i}$ is a homeomorphism which takes $V_{i}$ onto an unknotted solid torus $W$, the core of $S^{3}-$ int $W$, and a loop obtained by pushing a component of $h_{i}\left(F \cap T_{i}\right)$ into $S^{3}-$ int $W$. Since $E\left(L_{i}\right) \cap\left(S^{3}-\right.$ int $\left.W\right)$ is a 2-fold composing space, $\left.h_{i} \circ f\right|_{N_{i}} \circ h_{i}^{-1}$ extends to an involution of $E\left(L_{i}\right)$ with fixed point set a closed surface $F_{i}$ which meets the 2-fold composing space $E\left(L_{i}\right) \cap\left(S^{3}-\right.$ int $\left.W\right)$ in an essential annulus.

Lemma 3.1 implies that $\mathcal{T}_{w}$ contains at least one torus in case (2a) or (2b). Since the induction hypothesis implies that each $L_{i}$ has at least $2 g\left(F_{i}\right)$ components, $L$ has at least $2(1-d)+\sum_{i=1}^{d} 2 g\left(F_{i}\right)$ components. Moreover, since $F$ intersects $N_{0}$ in a system of essential annuli, the Euler characteristic of $F$ is $\chi(F)=\sum_{i=1}^{d} \chi\left(F_{i}\right)$. 
Then $g(F)=1-d+\sum_{i=1}^{d} g\left(F_{i}\right)$ and therefore $L$ has at least $2 g(F)$ components. Hence, the proof is complete.

REMARK 3.2. Theorem 1.1 is true even if the fixed point set $F$ is a surface with nonempty boundary. This is verified as follows. Using the technique presented for case (1) in the proof of Theorem 1.1, construct a satellite $L^{\prime}$ of $L$ by gluing 2 -fold composing spaces to $E(L)$ along boundary components of $E(L)$ which meet $F$. Then the involution $f$ of $E(L)$ extends to an involution $f^{\prime}$ of $E\left(L^{\prime}\right)$ with fixed point set a closed surface $F^{\prime}$ obtained by gluing annuli to $F$ along the boundary. Denote by $b(F)$ the number of boundary components of $F$. Then $2 g(F)=2 g\left(F^{\prime}\right)-b(F)$. Theorem 1.1 implies that $L^{\prime}$ has at least $2 g\left(F^{\prime}\right)$ components. Since $L^{\prime}$ is obtained from $L$ by adding $b(F)$ components, $L$ has at least $2 g(F)$ components.

\section{References}

[1] G. Burde and K. Murasugi, 'Links and Seifert fiber spaces’, Duke Math. J. 37 (1970), 89-93.

[2] J. A. Hillman, 'Symmetries of knots and links, and invariants of abelian coverings (Part II)', Kobe J. Math. 3 (1986), 149-165.

[3] T. Ikeda, 'Atoroidal decompositions of link exteriors', Kobe J. Math. 9 (1992), 71-88.

[4] T. Ikeda, 'Essential surfaces in graph link exteriors', Canad. Math. Bull. 52 (2009), 257-266.

[5] T. Ikeda, 'Aspherical symmetries of graph links', J. Knot Theory Ramifications, to appear.

[6] W. Jaco and P. Shalen, Seifert Fibered Spaces in 3-Manifolds, Memoirs of the American Mathematical Society, 220 (American Mathematical Society, Providence, RI, 1979).

[7] W. Jaco, Lectures on Three-Manifold Topology, Regional Conference Series in Mathematics, 43 (American Mathematical Society, Providence, RI, 1980).

[8] K. Johannson, Homotopy Equivalences of 3-Manifolds with Boundaries, Lecture Notes in Mathematics, 761 (Springer, Berlin, 1979).

[9] W. H. Meeks and P. Scott, 'Finite group actions on 3-manifolds', Invent. Math. 86 (1986), 287-346.

TORU IKEDA, Department of Mathematics, Faculty of Science, Kochi University, 2-5-1 Akebono-Cho, Kochi 780-8520, Japan

e-mail: ikedat@kochi-u.ac.jp 